\title{
Remarks on Bivariant Constructible Functions
}

\author{
Jean-Paul Brasselet \\ Shoji Yokura
}

Vienna, Preprint ESI 614 (1998)

May 21, 1999

Supported by Federal Ministry of Science and Transport, Austria Available via http://www.esi.ac.at 


\title{
REMARKS ON BIVARIANT CONSTRUCTIBLE FUNCTIONS
}

\author{
Jean-Paul Brasselet and Shoji Yokura*
}

Dedicated to the memory of Professor Nobuo Sasakura

\section{INTRODUCTION}

The so-called Chern-Schwartz-MacPherson class (or transformation) is the unique natural transformation from the covariant functor of constructible functions to the integral homology covariant functor, satisfying a certain normalization condition (see [14], and also [3]. [10]. [20].) The bivariant theory has been introduced by W.Fulton and R.MacPherson [9], and they conjectured (or posed as a question) the existence of a Grothendieck transformation from the bivariant theory of constructible functions to the bivariant homology theory in the category of complex algebraic varieties, which specializes to the original Chern-Schwartz-MacPherson transformation. The conjecture has been solved by Brasselet for a certain reasonable category [2] (see also [19] and [24]). In this paper we report some consequences of this Brasselet's theorem, concerning bivariant constructible functions (i.e., constructible functions satisfying the local Euler condition) and some related results and we also pose some problems.

\section{$\S 1$ Constructible functions and Chern-Schwartz-MacPherson classes}

A constructible set of an analytic variety $X$ is obtained from analytic subvarieties of $X$ by a finite number of unions, intersections and complements. A constructible function on a compact complex analytic variety $X$ is an integer-valued function on $X, \alpha: X \rightarrow \mathbb{Z}$, such that for each integer $n, \alpha^{-1}(n)$ is a constructible set of $X$. We say that a cellular decomposition $(K)$ of $X$ is $\alpha$-adapted if $\alpha$ is constant on the interior of each cell $\sigma$ of $(K)$, the value beeing denoted by $\alpha(\sigma)$.

Let $\mathcal{F}(X)$ denote the abelian group of constructible functions on $X$. Any constructible function can be expressed as a (finite) linear combination of the characteristic functions $\mathbb{1}_{W}$ 's where $W$ are reduced and irreducible subvarieties of $X$. Clearly the correspondence $\mathcal{F}$ assigning to each variety $X$ the abelian group $\mathcal{F}(X)$ becomes a contravariant functor when we consider the usual (functional) pull-back $f^{*}: \mathcal{F}(Y) \rightarrow \mathcal{F}(X)$ for a morphism $f: X \rightarrow Y$; i.e., $f^{*}(\alpha)(x):=\alpha(f(x))$. An interesting feature of the correspondence $\mathcal{F}$ is that it can be made a covariant functor when we consider the following pushforward:

$$
f_{*}\left(\mathbb{1}_{W}\right)(y):=\chi\left(f^{-1}(y) \cap W\right)
$$

(*) Partially supported by Grant-in-Aid for Scientific Research (No.10640084), the Japanese Ministry of Education, Science and Culture 
which is linearly extended with respect to the generators $\mathbb{1}_{W}$. Here $\chi(F)$ denotes the topological Euler-Poincaré characteristic of the space $F$. The proof of the covariant functoriality of $\mathcal{F}$ requires a stratification of the morphism $f$ (see [14], [21]).

Deligne and Grothendieck (in 1969) conjectured the following in the algebraic category:

Let $\mathcal{F}$ be the above covariant functor of constructible functions and

$H_{*}(: \mathbb{Z})$ be the usual $\mathbb{Z}$-homology covariant functor. Then there exists a unique natural transformation

$$
C_{*}: \mathcal{F} \rightarrow H_{*}(: \mathbb{Z})
$$

such that (normalization condition) if $X$ is smooth, then

$$
C_{*}\left(1_{X}\right)=c\left(T_{X}\right) \cap[X],
$$

where $c\left(T_{X}\right)$ is the total Chern cohomology class of the tangent bundle $T_{X}$ and $[X]$ is the fundamental homology class of $X$.

The conjecture was solved by MacPherson [14] (in 1974), using Chern-Mather classes, local Euler obstructions (which are constructible functions) and graph construction method. The folklore was that the above conjecture or theorem now was true in the analytic category also, and indeed in the analytic category MacPherson's proof works mutatis mutandis, except for the analyticity of the graph construction. However this analyticity was finally resolved affirmatively by M. Kwieciński in his thesis [13]. Thus the Chern-Schwartz-MacPherson transformation $C_{*}: \mathcal{F} \rightarrow H_{*}(: \mathbb{Z})$ can be considered in both the algebraic and analytic categories. The total homology class $C_{*}\left(\mathbb{1}_{X}\right)$ is called the Chern-Schwartz-MacPherson class of $X$. To avoid some possible confusion, we call the above transformation $C_{*}$ the Chern-SchwartzMacPherson transformation, emphasizing that it is a transformation. In fact, before the above conjecture was made M.-H.Schwartz [20] had already constructed characteristic cohomology classes of a (possibly singular) analytic variety embedded in a complex manifold, using the notion of radial vector field. For a given embedding $X$ in a manifold $M$ the Schwartz classes lie in $H_{X}^{*}(M)=H^{*}(M, M-X)$. It turned out that they are isomorphic to MacPherson's classes via Alexander duality isomorphism (see $[3]$ ).

\section{§2 Bivariant theory of CONSTRUCTIBLE FunCtions}

Let $\alpha$ be a constructible function on $X$. For $A \subset X$, we define

$$
\chi(A ; \alpha)=\sum_{n \in \mathbb{Z}} n \chi\left(A \cap \alpha^{-1}(n)\right),
$$

which is the Euler-Poincaré characteristic of A weighted by a ("pon- dérée par $\alpha$ ") ([2], [14], [19]). With this notation, the pushforward $f_{*} \alpha$ of the constructible function $\alpha$ under a morphism $f: X \rightarrow Y$ is expressed as follows:

$$
\left(f_{*} \alpha\right)(y):=\chi\left(f^{-1}(y) ; \alpha\right),
$$

i.e., the Euler-Poincaré characteristic of the fiber $f^{-1}(y)$ weighted by $\alpha$. Put it in another way, using the Chern-Schwartz-MacPherson transformation $C_{*}$, it can be rewritten as follows:

$$
\left(f_{*} \alpha\right)(y)=\int_{f^{-1}(y)} C_{*}\left(\left.\alpha\right|_{f^{-1}(y)}\right),
$$


the degree of the 0-dimensional component of the total Chern-Schwartz-MacPherson class of the constructible function $\left.\alpha\right|_{f^{-1}(y)}$ on the fiber $f^{-1}(y)$. This simple interpretation leads us to a naive question of what one could say about these classes $C_{*}\left(\left.\alpha\right|_{f^{-1}(y)}\right)$ parameterized by the target variety $Y$. It turns out that for this we need the bivariant theory of constructible functions which has been introduced by Fulton and MacPherson [9].

For a technical reason, the category which we treat is the following one, denoted by $\mathcal{S C}$ :

(i) The objects $\mathcal{O} b j(\mathcal{S C})$ consist of compact complex analytic varieties which are embeddable into smooth manifolds, and

(ii) The morphisms $\mathcal{H}_{\operatorname{sic}}(X, Y)$ consist of analytic maps $f: X \rightarrow Y$ which are cellular, i.e., with $(K)$ and $(L)$ being cellular decompositions of $X$ and $Y$ respectively, the image of each cell of $(K)$ is a cell of $(L)$ and the restriction of $f$ to the interior of each cell is constant rank.

At the moment it is not known whether any analytic map is cellular. Conjecturally it would be so.

In the following "cell" will always mean a closed cell, the interior of $\sigma$ will be denoted by $\sigma^{\circ}$ and we define the star $S t^{\circ} \sigma$ as the set of cells which meet the interior of $\sigma$.

In this category, the pushforward can be written as follows: Given cellular decompositions $(K)$ and $(L)$ of $X$ and $Y$ respectively, such that $(K)$ is $\alpha$-adapted and $f$ cellular, then

$$
\left(f_{*} \alpha\right)(y)=\sum_{\sigma \cap f^{-1}(y) \neq \phi}(-1)^{\operatorname{dim}_{f} \sigma} \alpha(\sigma)
$$

where $\operatorname{dim}_{f} \sigma$ denotes the relative dimension of $\sigma \in(K)$. Here we note that the above formula (2.1.1) is due to the fact that the topological Euler-Poincaré characteristic of a CW-complex can be also defined to be the alternating sum of the number of cells of a (in fact, any) cellular decomposition of the CW-complex, and therefore that the Euler-Poincaré characteristic weighted by $\alpha$ is equal to the alternating sum of the number of cells multiplied by the weights " $\alpha$ ".

Definition (2.1). Let $\alpha$ be a constructible function on $X$ and let $f: X \rightarrow Y$ be an analytic map. We say that $\alpha$ satisfies the local Euler condition with respect to $f$ if for any cellular decompositions $(K)$ and $(L)$ of $X$ and $Y$ respectively, such that $(K)$ is $\alpha$-adapted and $f$ is cellular, and if for any $x \in X, x \in \sigma^{\circ}, \sigma \in(K)$, the following equality holds

$$
\alpha(x)=\chi\left(S t^{o} \sigma \cap f^{-1}(y) ; \alpha\right)
$$

where $y \in S t^{\circ} f(\sigma)$ is arbitrary.

Using the values of $\alpha$ on the cells of $(K)$, the previous formula can be written

$$
\alpha(x)=\sum_{\substack{\sigma^{\prime} \subset S t^{\circ} \sigma \\ \sigma^{\prime} \cap f^{-1}(y) \neq \phi}}(-1)^{\operatorname{dim}_{f} \sigma^{\prime}} \alpha\left(\sigma^{\prime}\right)
$$

Remark 2.2. There is another definition of local Euler condition without refering to the cellular decomposition of a morphism (see [19]): $\alpha \in \mathcal{F}(X)$ satisfies the local 
Euler condition with respect to $f$ if for any point $x \in X$ and any local embedding $(X, x) \rightarrow\left(\mathbf{C}^{N}, 0\right)$ the following equality holds

$$
\alpha(x)=\chi\left(B_{\epsilon} \cap f^{-1}(z) ; \alpha\right),
$$

where $B_{\epsilon}$ is a sufficiently small open ball of the origin 0 with radius $\epsilon$ and $z$ is any point close to $f(x)$.

Definition (2.3). The bivariant group of constructible functions is defined, for every morphism $f: X \rightarrow Y$, by:

$$
\begin{gathered}
\mathbb{F}(X \stackrel{f}{\rightarrow} Y):=\{\alpha \in \mathcal{F}(X) \mid \alpha \text { satisfies the local Euler condition } \\
\text { with respect to } f\} .
\end{gathered}
$$

¿From this definition we see that

$$
\mathbb{F}(X \stackrel{i d}{\longrightarrow} X)=\{\alpha \in \mathcal{F}(X) \mid \alpha \text { is locally constant }\} .
$$

This fact will be used later.

For simplicity a constructible function satisfying the local Euler condition shall be called a bivariant constructible function. If $\mathbb{1}_{X}$ satisfies the local Euler condition with respect to the morphism $f: X \rightarrow Y$, i.e., $\mathbb{1}_{X} \in \mathbb{F}(X \stackrel{f}{\longrightarrow} Y)$, then the morphism $f$ is called an Euler morphism.

We can define the following three basic operations on $\mathbb{F}(X \rightarrow Y)$, which are called bivariant operations.

(BO-I) (Product operations): For morphisms $f: X \rightarrow Y$ and $g: Y \rightarrow Z$, the product operation

$$
\odot: \mathbb{F}(X \stackrel{f}{\longrightarrow} Y) \otimes \mathbb{F}(Y \stackrel{g}{\longrightarrow} Z) \rightarrow \mathbb{F}(X \stackrel{g f}{\longrightarrow} Z)
$$

is defined, for $\alpha \in \mathbb{F}(X \stackrel{f}{\longrightarrow} Y)$ and $\beta \in \mathbb{F}(Y \stackrel{g}{\longrightarrow} Z)$, by:

$$
(\alpha \odot \beta)(x):=\alpha(x) \cdot \beta(f(x)),
$$

i.e., $\alpha \odot \beta:=\alpha \cdot f^{*} \beta$. (To avoid some confusion, the symbol $\odot$ is used.)

(BO-II) (Pushforward operations): For morphisms $f: X \rightarrow Y$ (proper) and $g: Y \rightarrow$ $Z$, the pushforward operation

$$
f_{*}: \mathbb{F}(X \stackrel{g f}{\rightarrow} Z) \rightarrow \mathbb{F}(Y \stackrel{g}{\rightarrow} Z)
$$

is defined, for $\alpha \in \mathbb{F}(X \stackrel{g f}{\longrightarrow} Z)$, by:

$$
\left(f_{*} \alpha\right)(y):=\chi\left(f^{-1}(y) ; \alpha\right),
$$

which is the same as one described in $\$ 1$.

(BO-III) (Pull-back operations): For a fiber square

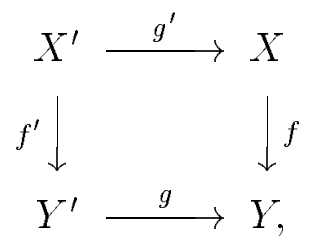


where $X^{\prime}=Y^{\prime} \times_{g=f} X$ is the fiber product and $f^{\prime}: X^{\prime} \rightarrow Y^{\prime}$ and $g^{\prime}: X^{\prime} \rightarrow X$ are the canonical projections, the pull-back operation

$$
g^{*}: \mathbb{F}(X \stackrel{f}{\longrightarrow} Y) \rightarrow \mathbb{F}\left(X^{\prime} \stackrel{g}{\longrightarrow} Y^{\prime}\right)
$$

is defined, for $\alpha \in \mathbb{E}(X \stackrel{f}{\longrightarrow} Y)$, by:

$$
g^{*} \alpha:=g^{\prime *} \alpha,
$$

which is the usual (functional) pull-bak.

It is known that these three operations are well-defined, and we give a proof of this fact for the sake of completeness.

Proof of well-definedness.

Let $(K),(L)$ and $(M)$ be any cellular decompositions of $X, Y$ and $Z$ respectively adapted to the corresponding constructible functions and such that the corresponding morphisms are cellular.

(BO-I): Let $x_{o}$ be a point of the interior of $\sigma_{o} \in(K)$. What we want to show is that

$$
\alpha \odot \beta\left(x_{o}\right)=\left(\alpha \cdot f^{*} \beta\right)\left(x_{o}\right)=\alpha\left(x_{o}\right) \beta\left(f\left(x_{o}\right)\right)=\chi\left(S t^{\circ} \sigma_{o} \cap(g \circ f)^{-1}(z) ; \alpha \odot \beta\right)
$$

where $z \in S t^{\circ}(g \circ f) \sigma_{o}$. We will denote by $y$ a point in $S t^{\circ} f\left(\sigma_{o}\right)$ and by $\tau_{o}=f\left(\sigma_{o}\right)$, so $z \in S t^{\circ} g\left(\tau_{o}\right)$. We start with the last term:

$$
\begin{aligned}
& \chi\left(S t^{o} \sigma_{o} \cap(g \circ f)^{-1}(z) ; \alpha \odot \beta\right) \\
& =\sum_{\substack{\sigma \subset S t^{\circ} \sigma_{o} \\
\sigma \cap(g \circ f)^{-1}(z) \neq \phi}}(-1)^{d i m_{f} \sigma} \alpha(\sigma) \cdot(-1)^{d i m_{g} f(\sigma)} \beta(f(\sigma)) \\
& =\sum_{\substack{\tau \subset S t^{\circ} \tau_{o} \\
\tau \cap g^{-1}(z) \neq \phi}}(-1)^{\operatorname{dim}_{g} \tau} \beta(\tau) \cdot \sum_{\substack{\tau=f(\sigma) \\
\sigma \subset S t^{\circ} \sigma_{o} \\
\sigma \cap f^{-1}(y) \neq \phi}}(-1)^{\operatorname{dim}_{f} \sigma} \alpha(\sigma) \\
& =\sum_{\substack{\tau \subset S t^{\circ} \tau_{o} \\
\tau \cap g^{-1}(z) \neq \phi}}(-1)^{\operatorname{dim}_{g} \tau} \beta(\tau) \cdot \chi\left(S t^{o} \sigma_{o} \cap f^{-1}(y) ; \alpha\right) \\
& =\beta\left(f\left(x_{o}\right)\right) \cdot \alpha\left(x_{o}\right) \\
& =\alpha \odot \beta\left(x_{o}\right) .
\end{aligned}
$$

$\square$ (BO-II): We must prove that, if $y \in \tau^{o}$ with $\tau \in(L)$, then

$$
\left(f_{*} \alpha\right)(y)=\chi\left(S t^{\circ} \tau \cap g^{-1}(z) ; f_{*} \alpha\right)
$$

for any $z \in S t^{\circ} g(\tau)$.

Denoting $h=g \circ f$, let us remark the following properties:

$$
f^{-1}\left(g^{-1}(z) \cap S t^{\circ} \tau\right)=\bigcup_{f(\sigma)=\tau} h^{-1}(z) \cap S t^{\circ} \sigma
$$

Let $\sigma^{\prime}$ be a cell of $(K)$ such that $f\left(\sigma^{\prime}\right) \subset S t^{\circ} \tau$, then

$$
A\left(\sigma^{\prime}, \tau\right)=\left\{\sigma \in(K) \mid \sigma^{\prime} \subset S t^{o} \sigma, f(\sigma)=\tau\right\},
$$


is the subset of the face $\sigma^{\prime} \cap f^{-1}(\tau)$ of $\sigma^{\prime}$ consisting of cells whose image is $\tau$. Its restriction to any fiber $f^{-1}(y), y \in \tau^{o}$ is a cell whose Euler-Poincaré characteristic is

$$
\chi\left(\sigma^{\prime} \cap f^{-1}(y)\right)=\sum_{\sigma \in A\left(\sigma^{\prime}, \tau\right)}(-1)^{\operatorname{dim}_{f} \sigma}=1 .
$$

This equality is a crucial observation which makes the proof of BO-II "the most fun" (cf $[9, \S 6.1 .2$, the last two lines of p. 61]). We have :

$$
\begin{aligned}
& \left(f_{*} \alpha\right)(y)=\chi\left(f^{-1}(y) ; \alpha\right) \\
& =\sum_{\sigma \cap f^{-1}(y) \neq \phi}(-1)^{\operatorname{dim}_{f} \sigma} \alpha(\sigma) \\
& =\sum_{\sigma \cap f^{-1}(y) \neq \phi}(-1)^{d i m_{f} \sigma} \sum_{\substack{\sigma^{\prime} \subseteq S t^{o} \sigma \\
\sigma^{\prime} \cap h^{-1}(z) \neq \phi}}(-1)^{d i m_{h} \sigma^{\prime}} \alpha\left(\sigma^{\prime}\right) \text { (by 2.1.2) } \\
& =\sum_{\substack{\sigma^{\prime} \subseteq S t^{\circ} \sigma \\
\sigma^{\prime} \cap h^{-1}(z) \neq \phi}}(-1)^{\operatorname{dim}_{h} \sigma^{\prime}}\left(\sum_{\sigma \in A\left(\sigma^{\prime}, \tau\right)}(-1)^{\operatorname{dim}_{f} \sigma}\right) \alpha\left(\sigma^{\prime}\right) \\
& =\sum_{\substack{\sigma^{\prime} \subset S t^{\circ} \sigma \\
\sigma^{\prime} \cap h^{-1}(z) \neq \phi}}(-1)^{\operatorname{dim}_{h} \sigma^{\prime}} \alpha\left(\sigma^{\prime}\right) \\
& =\sum_{\substack{\sigma^{\prime} \subset S t^{\circ} \sigma \\
\sigma^{\prime} \cap h^{-1}(z) \neq \phi}}(-1)^{\operatorname{dim}_{g} f\left(\sigma^{\prime}\right)}(-1)^{\operatorname{dim} \sigma_{f} \sigma^{\prime}} \alpha\left(\sigma^{\prime}\right) \\
& =\sum_{\substack{\tau^{\prime} \subset S t^{o} \tau \\
\tau^{\prime} \cap g^{-1}(z) \neq \phi}}(-1)^{\operatorname{dim}_{g} \tau^{\prime}}\left(\sum_{f\left(\sigma^{\prime}\right)=\tau^{\prime}}(-1)^{\operatorname{dim}_{f} \sigma^{\prime}} \alpha\left(\sigma^{\prime}\right)\right) \\
& =\sum_{\substack{\tau^{\prime} \subset S t^{o} \tau \\
\tau^{\prime} \cap g^{-1}(z) \neq \phi}}(-1)^{\operatorname{dim}_{g} \tau^{\prime}} \chi\left(f^{-1}\left(\tau^{\prime}\right) ; \alpha\right) \\
& =\sum_{\substack{\tau^{\prime} \subset S t^{o} \tau \\
\tau^{\prime} \cap g^{-1}(z) \neq \phi}}(-1)^{\operatorname{dim}_{g} \tau^{\prime}}\left(f_{*} \alpha\right)\left(\tau^{\prime}\right) \\
& =\chi\left(S t^{\circ} \tau \cap g^{-1}(z) ; f_{*} \alpha\right) .
\end{aligned}
$$

$\square$ (BO-III): Let $x^{\prime} \in \tau_{o}^{o}$ be a point in $X^{\prime}$. Then for any $y^{\prime} \in S t^{o} f^{\prime}\left(\tau_{o}\right)$ in $Y^{\prime}$, letting $\sigma_{o}=g^{\prime}\left(\tau_{o}\right)$ and $y=g\left(y^{\prime}\right)$, we have

$$
\begin{aligned}
\chi\left(S t^{o} \tau_{o} \cap f^{\prime-1}\left(y^{\prime}\right) ; g^{*} \alpha\right) & =\sum_{\substack{\tau \subset S t^{o} \tau_{o} \\
\tau \cap f^{\prime-1}\left(y^{\prime}\right) \neq \phi}}(-1)^{d i m_{f^{\prime}} \tau} g^{*} \alpha(\tau) \\
& =\sum_{\substack{\tau \subset S t^{o} \tau_{o} \\
\tau \cap f^{\prime-1}\left(y^{\prime}\right) \neq \phi}}(-1)^{d i m_{f^{\prime}} \tau} \alpha\left(g^{\prime}(\tau)\right) \\
& =\sum_{\substack{\sigma \subset S t^{o} \sigma_{o} \\
\sigma \cap f^{-1}(y) \neq \phi}}(-1)^{\operatorname{dim}_{f} \sigma} \alpha(\sigma) \\
& =\alpha\left(g^{\prime}\left(x^{\prime}\right)\right)=\left(g^{*} \alpha\right)\left(x^{\prime}\right) .
\end{aligned}
$$


$\square$ It is easy to see that these bivariant operations enjoy the following seven properties.

(B-1) Product is associative : for a diagram $X \stackrel{f}{\longrightarrow} Y \stackrel{g}{\longrightarrow} Z \stackrel{h}{\longrightarrow} W$ and $\alpha \in \mathbb{F}(X \stackrel{f}{\longrightarrow} Y), \beta \in \mathbb{F}(Y \stackrel{g}{\longrightarrow} Z)$ and $\gamma \in \mathbb{F}(Z \stackrel{h}{\longrightarrow} W)$,

$$
(\alpha \odot \beta) \odot \gamma=\alpha \odot(\beta \odot \gamma) \in \mathbb{F}(X \stackrel{\text { hgf }}{\longrightarrow} W) .
$$

(B-2) Pushforward is functorial: for a diagram $X \stackrel{f}{\longrightarrow} Y \stackrel{g}{\longrightarrow} Z \stackrel{h}{\longrightarrow} W$ and $\alpha \in$ $\mathbb{F}(X \stackrel{h g f}{\longrightarrow} W)$,

$$
(g f)_{*}(\alpha)=g_{*} f_{*}(\alpha) \in \mathbb{F}(Z \stackrel{h}{\rightarrow} W) .
$$

(B-3) Pullback is functorial: for a double fiber square

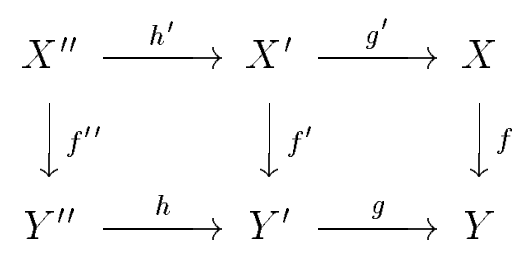

and $\alpha \in \mathbb{F}(X \stackrel{f}{\longrightarrow} Y)$,

$$
(g h)^{*}(\alpha)=h^{*} g^{*}(\alpha) \in \mathbb{F}\left(X^{\prime \prime} \stackrel{f^{\prime \prime}}{\rightarrow} Y^{\prime \prime}\right) .
$$

(B-4) Product and pushforward commute: for a diagram $X \stackrel{f}{\longrightarrow} Y \stackrel{g}{\longrightarrow} Z \stackrel{h}{\longrightarrow} W$ and $\alpha \in \mathbb{F}(X \stackrel{g f}{\longrightarrow} Z), \beta \in \mathbb{F}(Z \stackrel{h}{\longrightarrow} W)$,

$$
f_{*}(\alpha \odot \beta)=f_{*}(\alpha) \odot \beta \in \mathbb{F}(Y \stackrel{h g}{\rightarrow} W) .
$$

(B-5) Product and pullback commute: for a double fiber square

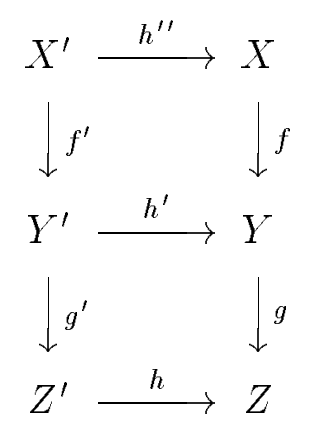

and $\alpha \in \mathbb{F}(X \stackrel{f}{\longrightarrow} Y), \beta \in \mathbb{F}(Y \stackrel{g}{\longrightarrow} Z)$,

$$
h^{*}(\alpha \odot \beta)=h^{\prime *}(\alpha) \odot h^{*}(\beta) \in \mathbb{F}\left(X^{\prime} \stackrel{g^{\prime} f^{\prime}}{\longrightarrow} Z^{\prime}\right) .
$$

(B-6) Pushforward and pullback commute: for a double fiber square

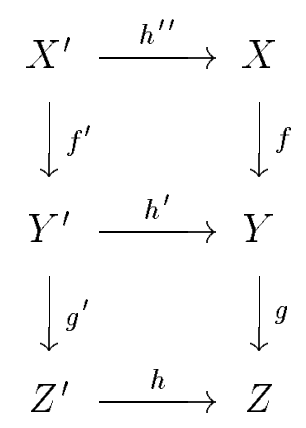


and $\alpha \in \mathbb{F}(X \stackrel{g f}{\longrightarrow} Z)$,

$$
f_{*}^{\prime}\left(h^{*}(\alpha)\right)=h^{*} f_{*}(\alpha) \in \mathbb{F}\left(Y^{\prime} \stackrel{g^{\prime}}{\longrightarrow} Z^{\prime}\right)
$$

(B-7) Projection formula: For a fiber square

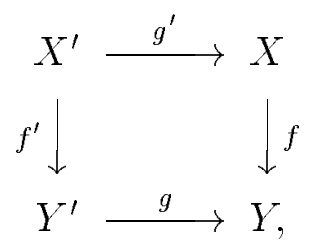

a morphism $Y \stackrel{h}{\longrightarrow} Z, \alpha \in \mathbb{F}(X \stackrel{f}{\longrightarrow} Y)$ and $\beta \in \mathbb{F}\left(Y^{\prime} \stackrel{h g}{\longrightarrow} Z\right)$.

$$
g_{*}^{\prime}\left(\left(g^{*} \alpha\right) \odot \beta\right)=\alpha \odot g_{*}(\beta) \in \mathbb{F}(X \stackrel{h f}{\rightarrow} Z) .
$$

Before finishing this section we note that the well-definedness of the pushforward (BO-II) implies the following

Proposition (2.4). Let $\alpha \in \mathbb{F}(X \stackrel{f}{\longrightarrow} Y)$, then the pushforward $f_{*} \alpha$ is a locally constant function on $Y$.

This can be seen as follows: Consider the pushforward on the following diagram:

$$
X \stackrel{f}{\longrightarrow} Y \stackrel{i d}{\longrightarrow} Y
$$

Indeed, for $\alpha \in \mathbb{F}(X \stackrel{f}{\longrightarrow} Y)=\mathbb{F}(X \stackrel{\text { id } f}{\longrightarrow} Y)$

$$
f_{*} \alpha \in \mathbb{F}(Y \stackrel{i d}{\rightarrow} Y),
$$

which implies that $f_{*} \alpha$ is locally constant since

$$
\mathbb{F}(X \stackrel{i d}{\longrightarrow} X)=\{\alpha \in \mathcal{F}(X) \mid \alpha \text { is locally constant }\}
$$

In other words the local Euler condition posed on a constructible function may be a right local condition to guarantee such a strong requirement that the Euler-Poincaré characteristic of the fibers weighted by $\alpha$ are locally constant. This is certainly a strong requirement for a map.

\section{$\S 3$ Bivariant Chern classes}

In general, a bivariant theory $B$ on a category $\mathcal{C}$ to abelian groups is an assignment to each morphism

$$
X \stackrel{f}{\rightarrow} Y
$$

in the category $\mathcal{C}$ an abelian group

$$
B(X \stackrel{f}{\rightarrow} Y)
$$


which is equipped with the three basic operations such as in (BO-I, BO-II, BO-III) above and satisfy the seven properties as in (B-1)-(B-7).

Let $\mathbb{H}(X \rightarrow Y)$ be the bivariant homology theory (see [2] and [9]). For a morphism $f: X \rightarrow Y$ and for any integer $i, H^{i}(X \rightarrow Y):=H^{i+2 m}(Y \times M, Y \times M-\Phi(X))$, where $\phi: X \rightarrow M$ is an embedding into a smooth manifold of real dimension $2 m$ and $\Phi:=(f, \phi): X \rightarrow Y \times M$ is an embedding. The definition is independent of the embedding $\phi: X \rightarrow M$. Then as in the case of the bivariant constructible function theory the three basic bivariant operations can be defined for the bivariant homology theory, namely we have the following (for details see Fulton-MacPherson's book [9]): (BO-I: $\mathbb{H}$ ) (Product operations): For morphisms $f: X \rightarrow Y$ and $g: Y \rightarrow Z$, the product operation

$$
\odot_{\mathbb{H}}: \mathbb{H}(X \stackrel{f}{\longrightarrow} Y) \otimes \mathbb{H}(Y \stackrel{g}{\longrightarrow} Z) \rightarrow \mathbb{H}(X \stackrel{g f}{\longrightarrow} Z)
$$

is defined.

(BO-II: $\mathbb{H}$ ) (Pushforward operations): For morphisms $f: X \rightarrow Y$ (prop- er) and $g: Y \rightarrow Z$, the pushforward operation

$$
f_{*}: \mathbb{H}(X \stackrel{g f}{\longrightarrow} Z) \rightarrow \mathbb{H}(Y \stackrel{g}{\longrightarrow} Z)
$$

is defined.

(BO-III: $\mathbb{H})$ (Pull-back operations): For a fiber square

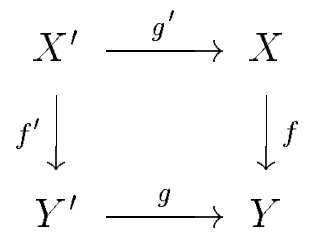

the pull-back operation

$$
g^{*}: \mathbb{H}(X \stackrel{f}{\longrightarrow} Y) \rightarrow \mathbb{H}\left(X^{\prime} \stackrel{g}{\longrightarrow} Y^{\prime}\right)
$$

is defined.

Fulton and MacPherson [9] conjectured (or posed as a question) the existence of a bivariant version of the Chern-Schwartz-MacPherson transformation $C_{*}: \mathcal{F} \rightarrow H_{*}($ : $\mathbb{Z}$ ), i.e., the existence of Grothendieck transformation (or "bivariant Chern class"), in the category of complex algebraic varieties. Brasselet [2] proved this conjecture in the category $\mathcal{S C}$ defined in $\S 2$. Also C. Sabbah [19] constructed a bivariant theory of cycles and J.Zhou [24] proved that Sabbah's bivariant Chern classes defined by bivariant cycles are the same as Brasselet's bivariant Chern classes.

Theorem (3.1). (Brasselet [2, III, Théorème]) Let $\mathcal{S C}$ be the category to be considered. There exists a Grothendieck transformation

$$
\gamma: \mathbb{F} \rightarrow \mathbb{H}
$$

such that if $X$ is a smooth variety, then

$$
\gamma\left(\mathbb{1}_{\pi}\right)=c(T X) \cap[X]
$$


where $\pi: X \rightarrow$ pt is a map to a point pt and $\mathbb{1}_{\pi}:=\mathbb{1}_{X} \in \mathbb{F}(X \stackrel{\pi}{\longrightarrow} p t)$. Namely, for each morphism $f: X \rightarrow Y, \gamma$ gives rise to a homomorphism

$$
\gamma: \mathbb{F}(X \stackrel{f}{\rightarrow} Y) \rightarrow \mathbb{H}(X \stackrel{f}{\rightarrow} Y)
$$

such that $\gamma$ preserves the basic three operations, i.e., (i) $\gamma(\alpha \odot \beta)=\gamma(\alpha) \odot \mathbb{H} \gamma(\beta)$, (ii) $\gamma\left(f_{*} \alpha\right)=f_{*} \gamma(\alpha)$ and (iii) $\gamma\left(f^{*} \alpha\right)=f^{*} \gamma(\alpha)$.

Remark 3.2. The uniqueness problem of $\gamma$ is still open. We will discuss it a little later in the next section (Remark (4.10)).

Brasselet constructs the above transformation in such a way that the ChernSchwartz-MacPherson classes $i_{y_{*}} C_{*}\left(\left.\alpha\right|_{f^{-1}(y)}\right)$ of the fibers weighted by $\alpha$ are locally constant, where $i_{y}: f^{-1}(y) \rightarrow X$ is the inclusion map. Of course this is a much stronger requirement than the local constancy of the Euler-Poincare characteristic of the fibers weighted by the constructible function $\alpha$. In fact as a consequence of the above Brasselet's theorem we can say more and we see that this quite strong requirement is a necessity for a bivariant constructible function.

Theorem (3.3). (1) Let $\alpha \in \mathbb{F}(X \stackrel{f}{\longrightarrow} Y)$ and let $V_{1}, V_{2}$ be subvarieties of $Y$ such that the Chern-Schwartz-MacPherson classes of $V_{1}$ and $V_{2}$ are homologous in $Y$, then the Chern-Schwartz-MacPherson classes $C_{*}\left(\left.\alpha\right|_{f^{-1}\left(V_{1}\right)}\right)$ and $C_{*}\left(\left.\alpha\right|_{f^{-1}\left(V_{2}\right)}\right)$ of the inverses $f^{-1}\left(V_{1}\right), f^{-1}\left(V_{2}\right)$ weighted with $\alpha$ are also homologous in $X$. Namely, if

$$
i_{1 *} C_{*}\left(V_{1}\right)=i_{2 *} C_{*}\left(V_{2}\right)
$$

with $i_{j}: V_{j} \rightarrow Y$ being the inclusion maps $(j=1,2)$, then

$$
e_{1 *} C_{*}\left(\left.\alpha\right|_{f^{-1}\left(V_{1}\right)}\right)=\epsilon_{2 *} C_{*}\left(\left.\alpha\right|_{f^{-1}\left(V_{2}\right)}\right)
$$

with $e_{j}: f^{-1}\left(V_{j}\right) \rightarrow X$ being the inclusion maps $(j=1,2)$.

(2) In particular, if $\alpha \in \mathbb{F}(X \stackrel{f}{\rightarrow} Y)$, then the Chern-Schwartz-Mac- Pherson classes $i_{y_{*}} C_{*}\left(\left.\alpha\right|_{f^{-1}(y)}\right)$ of the fibers weighted by are locally constant, where $i_{y}: f^{-1}(y) \rightarrow$ $X$ is the inclusion map.

Corollary (3.4). Let $f: X \rightarrow Y$ be an Euler morphism. Then if $V_{1}, V_{2}$ be subvarieties of $Y$ such that the Chern-Schwartz-MacPherson classes of $V_{1}$ and $V_{2}$ are homologous in $Y$, then the Chern-Schwartz-MacPherson classes $C_{*}\left(f^{-1}\left(V_{1}\right)\right)$ and $C_{*}\left(f^{-1}\left(V_{2}\right)\right)$ of the inverses

$f^{-1}\left(V_{1}\right), f^{-1}\left(V_{2}\right)$ are also homologous in $X$. Namely, if

$$
i_{1 *} C_{*}\left(V_{1}\right)=i_{2 *} C_{*}\left(V_{2}\right)
$$

with $i_{j}: V_{j} \rightarrow Y$ being the inclusion maps $(j=1,2)$, then

$$
e_{1 *} C_{*}\left(f^{-1}\left(V_{1}\right)\right)=\epsilon_{2 *} C_{*}\left(f^{-1}\left(V_{2}\right)\right)
$$

with $e_{j}: f^{-1}\left(V_{j}\right) \rightarrow X$ being the inclusion maps $(j=1,2)$. In particular, the Chern-Schwartz-MacPherson classes $i_{y_{*}} C_{*}\left(f^{-1}(y)\right)$ of the fibers are locally constant.

The proof of Theorem (3.3) goes as follows; for the sake of later use we give a detailed proof. 
Proof of Theorem (3.3). The constructible function $\alpha$ induces the following homomorphism,

$$
\alpha^{\mathbb{F}}: \mathcal{F}(Y) \rightarrow \mathcal{F}(X)
$$

defined by

$$
\alpha^{\mathbb{F}}(\beta):=\alpha \odot \beta=\alpha \cdot f^{*} \beta
$$

Then we can get the following commutative diagram:

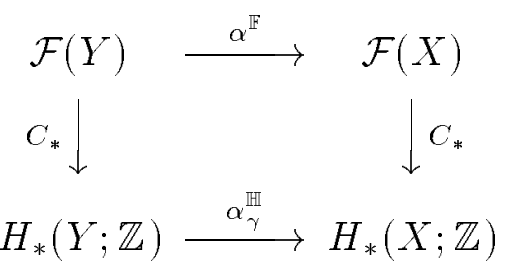

Here $\alpha_{\gamma}^{\mathbb{H I}}: H_{*}(Y ; \mathbb{Z})=\mathbb{H}(Y \rightarrow p t) \rightarrow H_{*}(X ; \mathbb{Z})=\mathbb{H}(X \rightarrow p t)$ is defined by

$$
\alpha_{\gamma}^{\mathbb{H}}(a):=\gamma(\alpha) \odot_{\mathbb{H}} a,
$$

where $\gamma: \mathbb{F} \rightarrow \mathbb{H}$ is a Grothendieck transformation and $\odot \mathbb{H}: \mathbb{H}(X \stackrel{f}{\rightarrow} Y) \otimes \mathbb{H}(Y \rightarrow$ $p t) \rightarrow \mathbb{H}(X \rightarrow p t)$ is the bivariant homology product operation. Here it should be noted that since the uniqueness of the Grothendieck transformation $\gamma$ is not known yet the homomorphism $\alpha_{\gamma}^{\text {HI }}$ could depend on the transformation $\gamma$ but that our statement is independent of the choice of $\gamma$. Of course the commutativity of the above diagram follows from the fact that the Grothendieck transformation preserves the three basic operations. First note that for a morphism $X \rightarrow p t$ the Grothendieck homomorphism $\gamma: \mathbb{F}(X \rightarrow p t) \rightarrow \mathbb{H}(X \rightarrow p t)$ is nothing but the Chern-SchwartzMacPherson transformation $C_{*}: \mathcal{F}(X) \rightarrow H_{*}(X ; \mathbb{Z})$. Then the commutativity can be seen as follows

$$
\begin{aligned}
C_{*} \alpha^{\mathbb{F}}(\beta) & =C_{*}(\alpha \odot \beta) \\
& =\gamma(\alpha \odot \beta) \\
& =\gamma(\alpha) \odot \mathbb{H} \gamma(\beta) \\
& =\gamma(\alpha) \odot \mathbb{H} C_{*}(\beta) \\
& =\alpha_{\gamma}^{\mathbb{H}} C_{*}(\beta) .
\end{aligned}
$$

We call the commutative diagram (3.3.1) a Verdier-type Riemann-Roch associated with the constructible function $\alpha$ (cf. [22]). To finish the proof of (1), we just apply this Verdier-type Riemann-Roch to two constructible functions $\mathbb{1}_{V_{1}}, \mathbb{1}_{V_{2}}$. First observe that for any subset $A \subset Y \alpha^{\mathbb{F}}\left(\mathbb{1}_{A}\right)=\alpha \cdot f^{*} \mathbb{1}_{A}=\alpha \cdot \mathbb{1}_{f^{-1}(A)}=\left.e_{*} \alpha\right|_{f^{-1}(A)}$, where $e: f^{-1}(A) \rightarrow X$ is the inclusion map. Now suppose that $V_{1}, V_{2}$ are subvarieties of $Y$ such that the Chern-Schwartz-MacPherson classes of $V_{1}$ and $V_{2}$ are homologous in $X$, i.e, $i_{1 *} C_{*}\left(V_{1}\right)=i_{2 *} C_{*}\left(V_{2}\right)$ with $i_{j}: V_{j} \rightarrow Y$ being the inclusion maps $(j=1,2)$. 
Then we have

$$
\begin{aligned}
\epsilon_{1 *} C_{*}\left(\left.\alpha\right|_{f^{-1}\left(V_{1}\right)}\right) & =C_{*}\left(\left.e_{1 *} \alpha\right|_{f^{-1}\left(V_{1}\right)}\right) \\
& =C_{*} \alpha^{\mathbb{F}}\left(\mathbb{1}_{V_{1}}\right) \\
& =\alpha_{\gamma}^{\mathbb{H}}\left(C_{*}\left(\mathbb{1}_{V_{1}}\right)\right) \\
& =\alpha_{\gamma}^{\mathbb{H}}\left(i_{1 *} C_{*}\left(V_{1}\right)\right) \\
& \left.=\alpha_{\gamma}^{\mathbb{H}}\left(i_{2 *} C_{*}\left(V_{2}\right)\right) \quad \text { (since } i_{1 *} C_{*}\left(V_{1}\right)=i_{2 *} C_{*}\left(V_{2}\right)\right) \\
& =\alpha_{\gamma}^{\mathbb{H}}\left(C_{*}\left(\mathbb{1}_{V_{2}}\right)\right) \\
& =C_{*} \alpha^{\mathbb{F}}\left(\mathbb{1}_{V_{2}}\right) \\
& =C_{*}\left(\left.e_{2 *} \alpha\right|_{f^{-1}\left(V_{2}\right)}\right) \\
& =e_{2 *} C_{*}\left(\left.\alpha\right|_{f^{-1}\left(V_{2}\right)}\right) .
\end{aligned}
$$

Thus (1) is proved and (2) is a special case of (1).

Remark (3.5). It follows from the definition of Eulerness that any local trivial fibration is always Euler. But Eulerness does not imply local triviality, as the following example (given by $\mathrm{T}$. Ohmoto) shows. Let $X=\left\{(x, y, z) \in \mathbb{C}^{3} \mid x^{2}+y^{2}+z=0\right\} \cup\{$ the $z$-axis $\}$ and let $f: X \rightarrow \mathbb{C}$ be the restriction to $X$ of the projection $p: \mathbb{C}^{3} \rightarrow \mathbb{C}$ to the third factor $\mathbb{C}$. The Milnor fiber at the origin is homotopic to the disjoint union of circle (i.e., the vanishing cycle) and one point, thus the Euler-Poincaré characteristic of a nearby fiber in a small neighborhood of the origin is equal to one. Hence at the origin it satisfies the local Euler condition, but it is not a local trivial fibration. At every point of $X$ off the origin the map $f$ is a local trivial fibration. Thus $f$ is Euler but not a local trivial fibration. The map $f$ is not proper, but the example can be modified into the following example of a map between compact varieties. Let us consider the following surface $E$ in $\mathbb{P}^{2} \times \mathbb{P}^{1}$ :

$$
\begin{aligned}
E:=\left\{\left(\left[x_{0}: x_{1}: x_{2}\right],\left[w_{0}: w_{1}\right]\right) \in \mathbb{P}^{2} \times \mathbb{P}^{1} \mid\right. & \\
& \left.w_{0} x_{0}^{2}+\left(w_{0}+w_{1}\right) x_{1}^{2}+w_{1} x_{2}^{2}=0\right\} .
\end{aligned}
$$

Let

$$
X:=E \cup\left([1: 0: 0] \times \mathbb{P}^{1}\right) \cup\left([0: 1: 0] \times \mathbb{P}^{1}\right) \cup\left([0: 0: 1] \times \mathbb{P}^{1}\right) .
$$

and let $f: X \rightarrow \mathbb{P}^{1}$ be the restriction to the subvariety $X$ of the projection $\mathbb{P}^{2} \times \mathbb{P}^{1} \rightarrow$ $\mathbb{P}^{1}$ to the second factor. Then just like the above example, at the three distinguished points $([1: 0: 0],[0: 1]),([0: 1: 0],[1:-1]),([0: 0: 1],[1: 0])$ the Milnor fiber of $f$ is homotopic to the union of the circle and one point and otherwise $f$ is locally trivial off these three points. Hence $f: X \rightarrow \mathbb{P}^{1}$ is Euler but not a local trivial fibration.

In general, some other well-studied morphisms, such as flat, open, $A_{f}$, and triangulable morphisms, are not Euler. For example, consider a Kodaira's elliptic surface [12], i.e., a surjective holomorphic map

$$
f: S \rightarrow C
$$

of a smooth compact complex surface $S$ onto a smooth compact complex curve $C$ such that its generic fiber is a smooth elliptic curve and that it has only finitely many singular fibers. This Kodaira elliptic surface $f: S \rightarrow C$ is not Euler, because the 
topological Euler-Poincaré characteristics of the fibers are not constant; the topological Euler-Poincaré characteristic of the generic fiber is zero but that of the singular fibers are not zero. On the other hand, it follows from [11, §4] that the map $f: S \rightarrow C$ is flat since $S$ and $C$ are smooth and (locally) the fibers have the same dimension (=1) and furthermore, since $C$ is smooth, hence Cohen-Macauley, $f$ is open due to the universal openness of the flat map. Since the target $C$ of the map $f$ is a smooth analytic curve, it follows from [11, Corollary 1, p.248] that $f$ is $A_{f}$, i.e., there exists a Whitney stratification of $f$ which satisfies Thom's $A_{f}$ condition. It is not clear whether it is triangulable or not, which is left for the reader. However, as an example of a morhism which is triangulable but not Euler, we can consider the following simple situation:

$$
X:=\left(\mathbb{P}^{1} \times[1: 0]\right) \cup\left([1: 0] \times \mathbb{P}^{1}\right) \subset \mathbb{P}^{1} \times \mathbb{P}^{1} .
$$

Namely, if we let $\left[z_{0}: z_{1}\right]$ and $\left[w_{0}: w_{1}\right]$ be the homogeneous coordinates of the first and second factor $\mathbb{P}^{1}$, respectively, then $X$ is defined by the equation $z_{1} w_{1}=0$. Let $f: X \rightarrow \mathbb{P}^{1}$ be the restriction of the projection $\mathbb{P}^{1} \times \mathbb{P}^{1} \rightarrow \mathbb{P}^{1}$ (either to the first factor or to the second factor) to the subvariety $X$. Then $f$ is obviously triangulable, but certainly not Euler because the topological Euler-Poincaré characteristics of the fibers are not constant; $\chi\left(f^{-1}([1: 0])\right)=\chi\left(\mathbb{P}^{1}\right)=2$ and $\chi\left(f^{-1}(x)\right)=1$ if $x \neq[1: 0]$.

At the moment a reasonable characterization of Eulerness is not known yet.

Before finishing this section we pose one problem. First, suggested by Proposition (2.4), one might be able to consider the following naïve group of constructible functions:

$$
\mathbb{F}^{l . c .}(X \stackrel{f}{\longrightarrow} Y):=\left\{\alpha \in \mathcal{F}(X) \mid f_{*} \alpha \text { is a locally constant function on } Y\right\}
$$

Then we can show the following

Proposition (3.6). Let us consider only topologically connected compact complex analytic varieties. Then the above naïve group of constructible functions $\mathbb{F}^{l . c .}(X \stackrel{f}{\longrightarrow}$ $Y)$ becomes a bivariant theory with the same operations as ones for $\mathbb{F}(X \stackrel{f}{\longrightarrow} Y)$.

Proof. We have only to show that the three operations are well-defined. First we note that since our varieties are assumed to be topologically connected, that $f_{*} \alpha$ is locally constant on $Y$ means that it is a constant function on $\mathrm{Y}$. This constancy is needed only for the well-definedness of the product operation, as we see below.

(1) (BO-I) (Product operations) For morphisms $f: X \rightarrow Y$ and $g: Y \rightarrow Z$, let $\alpha \in \mathcal{F}(X)$ such that $f_{*} \alpha$ is a constant function on $Y$ and $\beta \in \mathcal{F}(Y)$ such that $f_{*} \beta$ is a constant function on $Z$. Then we need to show that $(g f)_{*}(\alpha \odot \beta)$ is a constant function. First we note that $f_{*}(\alpha \odot \beta)=\left(f_{*} \alpha\right) \odot \beta$ by the commutativity of pushforward and product operation (B-4). Then since we can consider $f_{*} \alpha \in \mathbb{F}(Y \stackrel{i d}{\longrightarrow}$ $Y),\left(f_{*} \alpha\right) \odot \beta=\left(f_{*} \alpha\right) \cdot \beta=c \cdot \beta$, where $c=\chi\left(f^{-1}(y) ; \alpha\right)$ for any $y \in Y$ is a constant. Therefore $(g f)_{*}(\alpha \odot \beta)=g_{*}\left(f_{*}(\alpha \odot \beta)\right)=g_{*}(c \cdot \beta)=c \cdot g_{*}(\beta)$, which is a constant function because $g_{*}(\beta)$ is so. As we can see, the constancy of $f_{*} \alpha$ is crucial. (If it is not constant, we can easily get a counterexample.)

(2) (BO-II) (Pushforward operations) For morphisms $f: X \rightarrow Y$ and $g: Y \rightarrow Z$, and $\alpha \in \mathbb{F}^{l . c}(X \stackrel{g f}{\rightarrow} Z)$ we want to show that $f_{*} \alpha \in \mathbb{F}^{l . c}(Y \stackrel{g}{\rightarrow} Z)$. But this is obvious, because $(g f)_{*} \alpha=g_{*}\left(f_{*} \alpha\right)$. 
(3) (BO-III) (Pull-back operations) For a fiber square

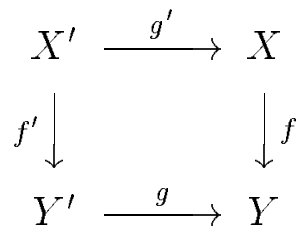

and $\alpha \in \mathcal{F}(X)$, we need to show that if $f_{*} \alpha$ is locally constant on $Y$, then $f_{*}^{\prime} g^{\prime *} \alpha$ is locally constant. For this we can use the following lemma ([5, Proposition 3.5]):

Lemma (3.6.1). The following diagram is commutative:

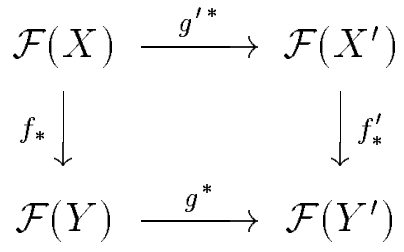

Since $f_{*} \alpha$ is locally constant, $g^{*} f_{*} \alpha$ is also locally constant. Then using the lemma, $g^{*} f_{*} \alpha=f_{*}^{\prime} g^{\prime *} \alpha$ is locally constant, thus $g^{*} \alpha:=g^{\prime *} \alpha \in \mathbb{F}^{l . c .}\left(X^{\prime} \stackrel{f^{\prime}}{\longrightarrow} Y^{\prime}\right)$.

Note that

$$
\begin{aligned}
& \mathbb{F}^{\text {l.c. }}(X \rightarrow p t)=\mathbb{F}(X \rightarrow p t)=\mathcal{F}(X), \\
& \begin{aligned}
\mathbb{F}^{\text {l.c. }}(X \stackrel{\text { id }}{\longrightarrow} X) & =\mathbb{F}(X \stackrel{\text { id }}{\longrightarrow} X) \\
& =\{\alpha \in \mathcal{F}(X) \mid \alpha \text { is locally constant on } X\},
\end{aligned}
\end{aligned}
$$

and

(3) in general, $\mathbb{F}(X \rightarrow Y) \subset \mathbb{F}^{l . c}(X \rightarrow Y)$ and they are not necessarily equal as the following example shows: (Example) Let $L_{1}$ be the diagonal of the cartesian product $\mathbb{P}^{1} \times \mathbb{P}^{1}$ of the 1 -dimensional projective space $\mathbb{P}^{1}$. Choose a point $z_{0}$ in $\mathbb{P}^{1}$, and consider another line $L_{2}:\left\{\left(z, z_{0}\right) \mid z \in P^{1}\right\} \subset \mathbb{P}^{1} \times \mathbb{P}^{1}$. Set $L:=L_{1} \cup L_{2} \subset \mathbb{P}^{1} \times \mathbb{P}^{1}$. Let $E$ be a smooth elliptic curve, so that its Euler characteristic $\chi(E)=0$. Let $X:=L \times E$. And let $f: X \rightarrow \mathbb{P}^{1}$ be the composite of the inclusion $X=L \times E \rightarrow$ $\mathbb{P}^{1} \times \mathbb{P}^{1} \times E$, the projection to the first two factors $\mathbb{P}^{1} \times \mathbb{P}^{1} \times E \rightarrow \mathbb{P}^{1} \times \mathbb{P}^{1}$ and the projection to the first factor $\mathbb{P}^{1} \times \mathbb{P}^{1} \rightarrow \mathbb{P}^{1}$. Then the Euler-Poincaré characteristic of the fibers are clearly locally constant; in fact $\chi\left(f^{-1}(z)\right)=0$ for any point $z$, which comes from the fact that $\chi(E)=0$. Thus the pushforward $f_{*} \mathbb{1}_{X}$ is locally constant. However, it is easy to see that the map $f$ is not Euler, i.e., $\mathbb{1}_{X} \notin \mathbb{F}(X \stackrel{f}{\rightarrow} Y)$. Because at every point of the fiber $f^{-1}\left(z_{0}\right)=\left\{\left(z_{0}, z_{0}\right)\right\} \times E, \mathbb{1}_{X}$ does not satisfy the local Euler condition with respect to $f$.

Let $\iota: \mathbb{F} \rightarrow \mathbb{F}^{l . c .}$ be the inclusion, i.e., $\iota(\alpha)=\alpha$.

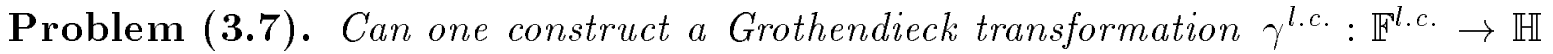
such that (1) $\gamma^{\text {l.c. }}\left(1_{\pi}\right)=c\left(T_{X}\right) \cap[X]$ if $X$ is smooth and (2) $\gamma=\gamma^{\text {l.c. }} \circ \iota$ ? 


\section{$\S 4$ Generalized Milnor numbers}

Definition (4.1). ( Parusiński's generalized Milnor number)([15], [16] and [17]) Let $X$ be a local complete intersection variety of a smooth variety $M$. Let $n=\operatorname{dim} X$. Then the Parusinski's generalized Milnor number $\mu(X)$ is defined to be

$$
\mu(X):=(-1)^{n+1}\left[\chi(X)-\int_{X} C^{F J}(X)\right]
$$

where $C^{F J}(X)$ is Fulton-Johnson's Chern class, defined to be $c\left(\left.T M\right|_{X}-N_{X} M\right) \cap[X]$ with $N_{X} M$ being the normal bundle of $X$. Note that this class is independent of the embedding of $X$ into a smooth variety $M$ (see Fulton's book [7, Example 4.2.6]).

Since $\chi(X)=\int_{X} C_{*}(X)$, as a simple generalization of the generalized Milnor number we have the following

Definition (4.2). (see [1], [4], [18], [23]) Let the situation be as in Definition (4.1) . The Milnor class $\mathcal{M}(X)$ is defined by

$$
\mathcal{M}(X):=(-1)^{n+1}\left[C_{*}(X)-C^{F J}(X)\right]
$$

With these definitions we can show the following theorem.

Theorem (4.3). Let $f: X \stackrel{r}{\longrightarrow} M \stackrel{p}{\longrightarrow} Y$ be an Euler and local complete intersection morphism (i.e., $r: X \rightarrow M$ is a regular embedding and $p: M \rightarrow Y$ is a smooth morphism) such that over each point $y \in Y$, the restriction to the fibers $r_{y}: X_{y} \rightarrow M_{y}$ is also a regular embedding with $\operatorname{dim} X_{y}=n$, i.e., the fiber $X_{y}:=f^{-1}(y)$ is a local complete intersection variety of the smooth fiber $M_{y}:=p^{-1}(y)$. Then the Milnor class $\mathcal{M}\left(X_{y}\right)$ of the fiber $X_{y}$ (considered as classes of $X$ ) are locally constant. In particular, the generalized Milnor number of the fibers $X_{y}$ are locally constant.

Proof. Firstly we remark that the smoothness of the fiber $M_{y}$ comes from the smoothness of the morphism $p$. Since $f: X \rightarrow Y$ is a local complete intersection morphism, we can define the following homomorphism

$$
c\left(T_{f}\right) \cap f^{*}: H_{*}(Y ; \mathbb{Z}) \rightarrow H_{*}(X ; \mathbb{Z})
$$

where $T_{f}$ is the virtual relative tangent bundle, defined to be

$$
T_{f}:=i^{*} T_{p}-N_{X} M
$$

and $f^{*}: H_{*}(Y ; \mathbb{Z}) \rightarrow H_{*}(X ; \mathbb{Z})$ is the Gysin homomorphism [7, Example 19.2.1]. Since we are in the homology theory, the homology classes $c\left(T_{f}\right) \cap f^{*}([y])$ are certainly locally constant. Since $f: X \rightarrow Y$ is also Euler, it follows from Corollary (3.4) that the Chern-Schwartz-MacPherson classes $i_{y_{*}} C_{*}\left(X_{y}\right)$ of the fibers $X_{y}$ are locally constant. So to prove the theorem we only need to prove the following equality

$$
c\left(T_{f}\right) \cap f^{*}([y])=i_{y_{*}} C^{F J}\left(X_{y}\right)
$$


for which we proceed as follows:

$$
\begin{aligned}
& c\left(T_{f}\right) \cap f^{*}([y])=c\left(T_{f}\right) \cap i^{*}\left(\left[M_{y}\right]\right) \\
& =c\left(T_{f}\right) \cap i^{*} i_{y_{*}}\left(\left[M_{y}\right]\right) \\
& \left.=c\left(T_{f}\right) \cap i_{y_{*}} i^{*}\left(\left[M_{y}\right]\right) \quad \text { (by }[5 \text {, Theorem }(6.2)(\mathrm{a})]\right) \\
& \left.=i_{y_{*}}{ } \mid c\left(i_{y}{ }^{*} T_{f}\right) \cap i^{*}\left(\left[M_{y}\right]\right)\right) \text { (by the projection formula) } \\
& \left.\left.=i_{y_{*}}{ } \mid c\left(T_{f_{y}}\right) \cap\left[X_{y}\right]\right)\right) \quad(\text { by }[5, \text { Example }(6.2 .1)]) \\
& =i_{y_{*}} C^{F J}\left(X_{y}\right) \text {. }
\end{aligned}
$$

Motivated by this result, we can consider the following: Since we are mostly interested in homology classes determined by subvarieties of a variety, we consider the Chow group $A(X)$, i.e., the group of cycles modulo rational equivalence [7], and the following homology group, which shall be provisionally called the "algebraic homology group", denoted by $A H_{*}(X ; \mathbb{Z})$ :

$$
A H_{*}(X ; \mathbb{Z}):=\text { Image }\left(c l: A(X) \rightarrow H_{*}(X ; \mathbb{Z})\right),
$$

where $c l: A(X) \rightarrow H_{*}(X ; \mathbb{Z})$ is the cycle map [6, $\left.\S 19.1\right]$.

Lemma (4.4). For a variety $X$,

$$
A H_{*}(X ; \mathbb{Z})=\operatorname{Image}\left(C_{*}: \mathcal{F}(X) \rightarrow H_{*}(X ; \mathbb{Z})\right)
$$

Proof. First of all we note that MacPherson's proof [14] actually shows that $C_{*}$ : $\mathcal{F}(X) \rightarrow H_{*}(X ; \mathbb{Z})$ is the composite of the homomorphism $C_{*}: \mathcal{F}(X) \rightarrow A(X)$ into the Chow homology group and the cycle map $c l: A(X) \rightarrow H_{*}(X ; \mathbb{Z})$. Here we use the same notation $C_{*}$, i.e.,

$$
C_{*}=\mathrm{cl} \circ C_{*}
$$

In fact it is easy to see by induction on dimension that the homomorphism $C_{*}$ : $\mathcal{F}(X) \rightarrow A(X)$ is always surjective, because for any subvariety $W C_{*}\left(\mathbb{1}_{W}\right)=[W]+$ lower classes. Thus we get $A H_{*}(X ; \mathbb{Z})=\operatorname{Image}\left(C_{*}: \mathcal{F}(X) \rightarrow H_{*}(X ; \mathbb{Z})\right)$.

Now consider a Verdier-type Riemann-Roch diagram associated with the bivariant constructible function $\mathbb{1}_{X}$ :

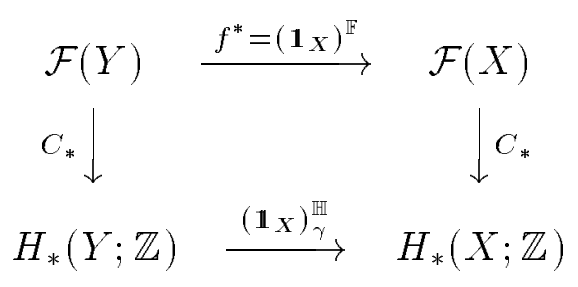

where $\gamma: \mathbb{F} \rightarrow \mathbb{H}$ is a Grothendieck transformation (cf. [22]). It follows from Lemma (4.4) that the restricted homomorphism $f^{E u}:=\left.\left(\mathbb{1}_{X}\right)_{\gamma}^{\mathbb{H}}\right|_{A H_{*}(Y ; \mathbb{Z})}: A H_{*}(Y ; \mathbb{Z}) \rightarrow A H_{*}(X ; \mathbb{Z})$ can be expressed as follows:

$$
f^{E u}:=C_{*} f^{*} C_{*}^{-1}: A H_{*}(Y ; \mathbb{Z}) \rightarrow A H_{*}(X ; \mathbb{Z}),
$$


which is well-defined because of the commutativity of the above Verdier-type Riemann -Roch diagram. And of course we have the following homomorphism

$$
c\left(T_{f}\right) \cap f^{*}: A H_{*}(Y ; \mathbb{Z}) \rightarrow A H_{*}(X ; \mathbb{Z})
$$

These two homomorphisms coincide when $f: X \rightarrow Y$ is a smooth morphism, but in general they are not identical if $f$ is not smooth $([22])$. So it is quite natural to pose the problem of describing the difference between the two.

Problem (4.5). Let $f: X \rightarrow Y$ be an Euler and local complete intersection morphism. Then give an explicit description of the following defect $\delta$ :

$$
f^{E u}=c\left(T_{f}\right) \cap f^{*}+\delta .
$$

Remark 4.6. For a hypersurface $X$ Parusiński and Pragacz [17] give an interesting and promising formula for the generalized Milnor number $\mu(X)$, in terms of the Chern-Schwartz-MacPherson of the closure of the strata of a Whitney stratification of $X$. Suggested by their result and Theorem (4.3), we expect that the defect $\delta$ in the above looked-for formula will be possibly described using a Whitney stratification of a morphism $f$. After the preparation of the manuscript we learned that in the hypersurface case the Parusiński-Pragacz's formula for the Milnor number $\mu(X)$ has been generalized to a formula for the Milnor class $\mathcal{M}(X)$ in [18].

An interesting feature of this looked-for formula is that it implies some relationship among Fulton-Johnson's canonical class

$\mathcal{C}(f):=c\left(T_{f}\right) \cap[X]$ of a local complete intersection morphism $f[8]$ and the ChernSchwartz-MacPherson class $C_{*}(X)$ of the source variety $X$ and (hopefully) some kind of invariants of singularities of the morphism $f$. Here is a citation from [8, p.382]: “... It would be interesting to relate the canonical class of a mapping to its singularities."

Proposition (4.7). Let $f: X \rightarrow Y$ be an Euler and local complete intersection morphism. Then we have the following formula:

$$
\mathcal{C}(f)=C_{*}(X)+\sum a_{V} C_{*}\left(f^{-1}(V)\right)-\delta([Y])
$$

where $[Y]=C_{*}(Y)+\sum_{\operatorname{dim} V<\operatorname{dim} Y} a_{V} C_{*}(V)$. In particular, if $f$ is smooth, then

$$
\mathcal{C}(f)=C_{*}(X)+\sum a_{V} C_{*}\left(f^{-1}(V)\right)
$$

Proof. First we observe that since

$$
[Y]=C_{*}(Y)+\sum_{\operatorname{dim} V<\operatorname{dim} Y} a_{V} C_{*}(V)
$$

we can take

$$
C_{*}^{-1}([Y])=\mathbb{1}_{Y}+\sum_{\operatorname{dim} V<\operatorname{dim} Y} a_{V} \mathbb{1}_{V} .
$$


Then

$$
\begin{aligned}
\mathcal{C}(f) & =c\left(T_{f}\right) \cap[X] \\
& =c\left(T_{f}\right) \cap f^{*}([Y]) \\
& =f^{E u}([Y])-\delta([Y]) \\
& =C_{*} f^{*} C_{*}^{-1}([Y])-\delta([Y]) \\
& =C_{*} f^{*}\left(\mathbb{1}_{Y}+\sum_{\operatorname{dim} V<\operatorname{dim} Y} a_{V} \mathbb{1}_{V}\right)-\delta([Y]) \\
& =C_{*}(X)+\sum_{\operatorname{dim} V<\operatorname{dim} Y} a_{V} C_{*}\left(f^{-1}(V)\right)-\delta([Y]) .
\end{aligned}
$$

Proposition (4.8). Let $f: X \rightarrow Y$ be an Euler morphism and let $Y$ be topologically connected. Then for any algebraic homology class $\alpha \in A H_{*}(Y ; \mathbb{Z})$ we have

$$
\int_{X} f^{E u}(\alpha)=\chi_{f} \cdot \int_{Y} \alpha .
$$

Here $\chi_{f}$ denotes the topological Euler-Poincaré characteristic of any fiber.

Proof. Since any homology class $\alpha \in A H_{*}(Y ; \mathbb{Z})$ is generated by the Chern-SchwartzMacPherson class $C_{*}(V)=C_{*}\left(\mathbb{1}_{V}\right)$ of subvarieties $V$, it suffices to show the formula for $\alpha=C_{*}\left(\mathbb{1}_{V}\right)$.

$$
\begin{aligned}
\int_{X} f^{E u}\left(C_{*}\left(\mathbb{1}_{V}\right)\right) & =\int_{Y} f_{*} f^{E u}\left(C_{*}\left(\mathbb{1}_{V}\right)\right) \\
& =\int_{Y} f_{*} C_{*} f^{*} C_{*}^{-1}\left(C_{*}\left(\mathbb{1}_{V}\right)\right) \quad\left(\text { since } f^{E u}=C_{*} f^{*} C_{*}^{-1}\right) \\
& =\int_{Y} C_{*} f_{*} f^{*}\left(\mathbb{1}_{V}\right) \quad\left(\text { since } f_{*} C_{*}=C_{*} f_{*}\right) \\
& =\int_{Y} C_{*}\left(\chi_{f} \cdot \mathbb{1}_{V}\right) \quad\left(f_{*} f^{*}\left(\mathbb{1}_{V}\right)=\chi_{f} \cdot \mathbb{1}_{V}\right) \\
& =\chi_{f} \cdot \int_{Y} C_{*}\left(\mathbb{1}_{V}\right) .
\end{aligned}
$$

\section{Problem (4.9).}

(1) Let $f: X \rightarrow Y$ be a local complete intersection morphism and let $Y$ be topologically connected. Let $\alpha \in A H_{*}(Y ; \mathbb{Z})$. Describe the following number as in the above proposition

$$
\int_{X} c\left(T_{f}\right) \cap f^{*}(\alpha) .
$$

(2) Let $f: X \rightarrow Y$ be a local complete intersection morphism with $Y$ being topologically connected such that $f: X \rightarrow M \rightarrow Y$ and that for each $y \in Y$ 
$r_{y}: X_{y} \rightarrow M_{y}$ is a regular embedding with $\operatorname{dim} X_{y}=n$, i.e., $X_{y}$ is a local complete intersection of the smooth fiber $M_{y}$. Then is it true that

$$
\int_{X} c\left(T_{f}\right) \cap f^{*}(\alpha)=\chi_{f}^{F J} \cdot \int_{Y} \alpha ?
$$

Here $\chi_{f}^{F J}=\int_{X_{y}} C^{F J}\left(X_{y}\right)$ is called the Fulton-Johnson's characteristic of the fiber.

If (2) of the above problem be true, then we will get the following formula:

$$
\int_{X} \delta(\alpha)=(-1)^{n+1} \mu_{f} \cdot \int_{Y} \alpha,
$$

where $\mu_{f}$ is the generalized Milnor number of the fiber.

Remark 4.10. Here we remark a little on the uniqueness problem of the bivariant Chern class. If we consider the algebraic homology group instead of the usual homology group, then to some extent we could see the "uniqueness" in the following sense. We want to see that if $\gamma, \gamma^{\prime}: \mathbb{F} \rightarrow \mathbb{H}$ are two Grothendieck-Chern transformations, then for any bivariant constructible function $\alpha$ the bivariant homology $\gamma(\alpha)=\gamma^{\prime}(\alpha)$. If we consider these two bivariant homology classes $\gamma(\alpha)$ and $\gamma^{\prime}(\alpha)$ as homological operators $\alpha_{\gamma}^{\mathbb{H}}(a)=\gamma(\alpha) \odot_{\mathbb{H}} a$ and $\alpha_{\gamma^{\prime}}^{\mathbb{H}}(a)=\gamma^{\prime}(\alpha) \odot_{\mathbb{H}} a$, which both define the homomorphism

$$
A H_{*}(Y ; \mathbb{Z}) \rightarrow A H_{*}(X ; \mathbb{Z})
$$

However, in the same argument as above we have the following equality:

$$
\alpha_{\gamma}^{\mathbb{H}}=C_{*} \alpha^{\mathbb{F}} C_{*}^{-1}=\alpha_{\gamma^{\prime}}^{\mathbb{H}} .
$$

Thus all the Grothendieck transformations induce the same homological operators if they are restricted to the algebraic homological classes. In particular, in the case when the cycle map $c l: A_{*}(X) \rightarrow H_{*}(X)$ is an isomorphism, e.g., if $X$ has a cellular decomposition (see [7, Example 1.9.1 and Example 19.1.11]), then the transformation $\gamma: \mathbb{F} \rightarrow \mathbb{H}$ is unique if it is considered as the homological operator $\alpha_{\gamma}^{\mathbb{H}}$. When the bivariant homology theory is replaced by the bivariant Chow homology theory ([6], $[7]$ ), see [6] for the uniqueness.

Acknowledgement. This work was partially processd during two stays of the first named author at University of Kagoshima, which he thanks for invitation. Also some of the work was done while the second named author was staying at the Erwin Schrödinger Institute, Vienna, Austria, in summer 1997. He wishes to express his thanks to the staff of the the institute, in particular the director, Peter W. Michor for their hospitality during his stay at the institute. And we also thank P. Aluffi, M. Kwieciński, T. Ohmoto, J. Zhou and the referee for their helpful comments.

\section{REFERENCES}

1. P. Aluffi, Chern classes for singular hypersurfaces, Trans. Amer. Math. Soc., (to appear).

2. J.P. Brasselet, Existence des classes de Chern en théorie bivariante, Astérisque 101-102 (1981), $7-22$. 
3. J.P. Brasselet and M.H. Schwartz, Sur les classes de Chern d'une ensemble analytique complexe, Astérisque 82-83 (1981), 93-148.

4. J.P. Brasselet, D. Lehmann, J. Seade and T. Suwa, Milnor classes of local complete intersections, Hokkaido Univ. Preprint Series in Math. \# 413 (1998).

5. L. Ernström, Topological Radon transforms and the local Euler obstruction, Duke Math. J. 76 (1994), 1-21.

6. Bivariant Schwartz-MacPherson classes with values in Chow groups, preprint (1994).

7. W. Fulton, Intersection Theory, Springer-Verlag, 1984.

8. W. Fulton and K. Johnson, Canonical classes on singular varieties, Manuscripta Math. 32 (1980), 381-389.

9. W. Fulton and R. MacPherson, Categorical frameworks for the study of singular spaces, Memoirs of Amer. Math.Soc. 243 (1981).

10. G. Gonzalez-Sprinberg, L'obstruction locale d'Euler et le théorème de MacPherson, Astérisque 82-83 (1981), 7-32.

11. H. Hironaka, Stratification and flatness, in Real and complex singularities, Oslo 1976, P. Holm (ed.), Sijthoff and Noordhoff (1977), 199-265.

12. K. Kodaira, On compact complex analytic surfaces, I, II, III, Ann. of Math., 71 (1960), 111152; 77 (1963), 563-626; 78 (1963), 1-40 78 (1963), 1-40.

13. M. Kwieciński, Sur le transformé de Nash et la construction du graph de MacPherson, in Thèse, Université de Provence (1994).

14. R. MacPherson, Chern classes for singular algebraic varieties, Ann.of Math. 100 (1974), 423432.

15. A. Parusiński, A generalization of the Milnor number, Math.Ann. 281 (1988), $247-254$.

16. Multiplicity of the dual variety, Bull.London Math.Soc. 23 (1991), 429-436.

17. A. Parusiński and P. Pragacz, A formula for the Euler characteristic of singular hypersurfaces, J.Algebraic Geometry 4 (1995), 337-351.

18. Characteristic classes of hypersurfaces and characteristic cycles, preprint (1998).

19. C. Sabbah, Espaces conormaux bivariants, Thèse, l'Université Paris VII (1986).

20. M.-H. Schwartz, Classes caractéristiques définies par une stratification d'une variété analytique complexe, C.R.Acad.Sci. Paris t.260 (1965), 3262-3264, 3535-3537.

21. J.-L. Verdier, Stratifications de Whitney et théorème de Bertini-Sard, Inventiones Math 36 (1976), 295-312.

22. S. Yokura, On a Verdier-type Riemann-Roch for Chern-Schwartz-Mac- Pherson class, Topology and Its Applications 95 (1999), (to appear).

23. _ On characteristic classes of complete intersections, to appear in "Algebraic Geometry - Hirzebruch 70", Contemporary Mathematics Amer. Math. Soc..

24. J. Zhou, Classes de Chern en théorie bivariante, in Thèse, Université Aix-Marseille II (1995).

J.-P.Brasselet: Institut de Mathématiques de Luminy, Luminy, Case 930, 13288 MarSeille Cedex 9, France

E-mail address: jpb@iml.univ-mrs.fr

S.Yokura: Department of Mathematics and Computer Science, Faculty of Science, University of Kagoshima, 1-21-35 Korimoto, Kagoshima 890-0065, Japan

E-mail address: yokura@sci.kagoshima-u.ac.jp 